\title{
Improved Design of an Ultra-Wideband Planar Slot Antenna
}

\author{
William O'Keefe Coburn \\ US Army Research Laboratory \\ Adelphi, MD 20783, USA \\ william.o.coburn.civ@mail.mil
}

\begin{abstract}
Planar antenna designs have many advantages such as low-profile, light-weight, and ease of fabrication and integration. Here, a planar slot antenna implemented on a thin substrate with coplanar waveguide feed is considered. This paper summarizes a numerical investigation using both frequency and time-domain solvers. The results serve to guide the future analysis of broadband antennas for ground penetrating radars.
\end{abstract}

\section{INTRODUCTION}

This paper presents a numerical analysis of an UltraWideband (UWB) planar slot antenna using FEKO (www.feko.info), based on the Method of Moments and GEMS (www.2comu.com), a Time-Domain Finite-Difference solver. The government code SENTRi (www.hpc.mil), based on the Finite Element Boundary Integral Method is also used.

The antenna is a coplanar waveguide (CPW) fed patch radiator exciting a rectangular slot. This structure has a larger impedance bandwidth (IBW) and higher gain compared to the radiator only as a planar monopole antenna. A metal ground plane can also be used to increase directivity, but the height must be larger than about 0.2 wavelengths and causes variations in the gain vs. frequency. For handheld Ground Penetrating Radar (GPR) applications a flat gain vs. frequency is desired. The embodiment considered here is restricted in size to less than 3inch square and minimum height. The boresight (i.e., at zenith) gain was improved at high frequency by using a slotted radiator.

\section{ANTENNA DESIGN}

A broadband planar slot can be obtained by careful design of the structure exciting the slot and tapering the slot corners. A common implementation was presented in [1] shown in Fig. 1 (a). The antenna size was increased by a factor of three and modified to improve the low frequency bandwidth as shown in Fig. 1 (b). The low frequency bandwidth was improved further by rounding the corners of the slot aperture and shown in Fig. 1 (c) with the fabricated antenna shown in Fig. 1 (d). The antenna is on RT/Duroid 5880 having $\varepsilon_{\mathrm{r}}=2.22$ and $\tan \delta=.0009$ with thickness $0.787 \mathrm{~mm}$ (31-mils) [2]. This planar slot was simulated with FEKO and SENTRi over the frequency range $0.3-4 \mathrm{GHz}$ with the $\mathrm{S}_{11}$ comparison shown in Fig. 2. The numerical results have similar frequency dependence and fair agreement in amplitude. But neither fully captures the measured impedance variations.

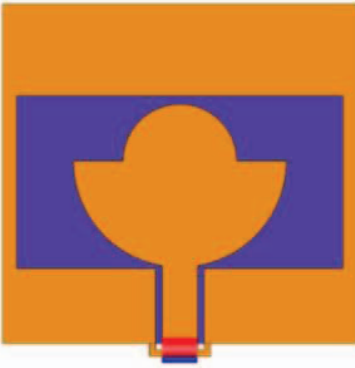

(a)

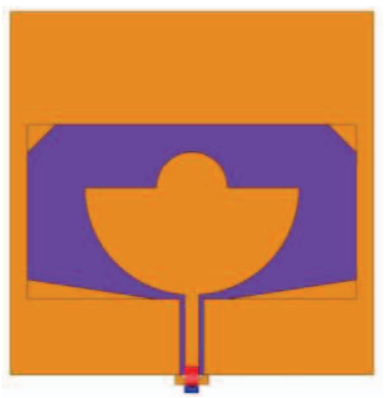

(c)

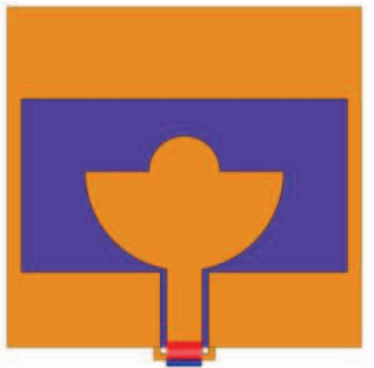

(b)

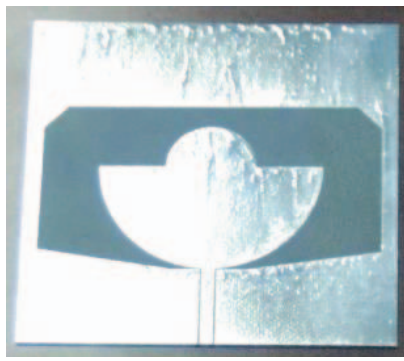

(d)
Fig. 1. Planar antennas: (a) original design [1], (b) modified version, (c) baseline design, and (d) fabricated antenna.

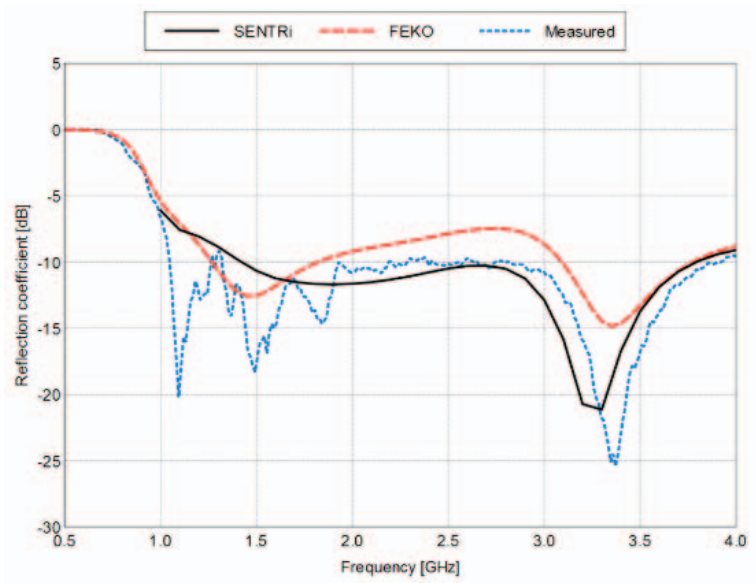

Fig. 2. Fabricated antenna reflection coefficient. 


\section{SLOtTEd RADiATOR MOdificAtion}

The antenna is modified with a shaped slot in the planar radiator to extend the IBW to lower frequencies and improve pattern stability at high frequency. The reflection coefficient comparison is shown in Fig. 3 (a). The peak gain tilts from zenith in the original design which is improved with the modified radiator as shown in Fig. 3 (b). The theta-polarized pattern cut along the CPW feed (E-plane) is shown in Fig. 4 at $4 \mathrm{GHz}$ indicating the beam tilting from zenith. The onset of this beam tilt is about $3 \mathrm{GHz}$ and becomes more pronounced with increasing frequency. Additional results with the available measured data will be included in the presentation. The results indicate that for GPR applications the antenna is poorly matched at low frequency. The antenna transient excitation and radiated field indicate some late time ringing which can be mitigated by absorber loading. This loading requires a tradeoff between impedance matching at low frequencies and antenna efficiency and should be measured to determine adequate radar performance.

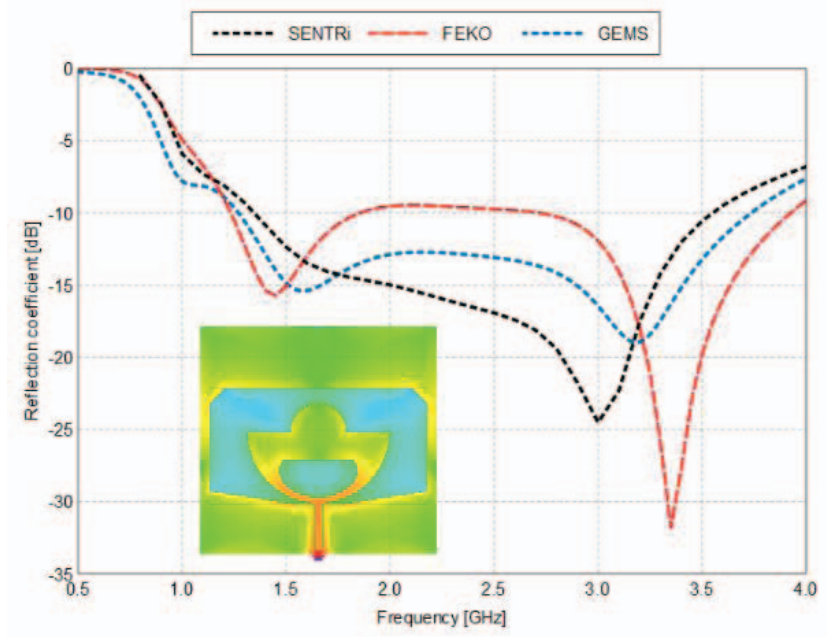

(a)

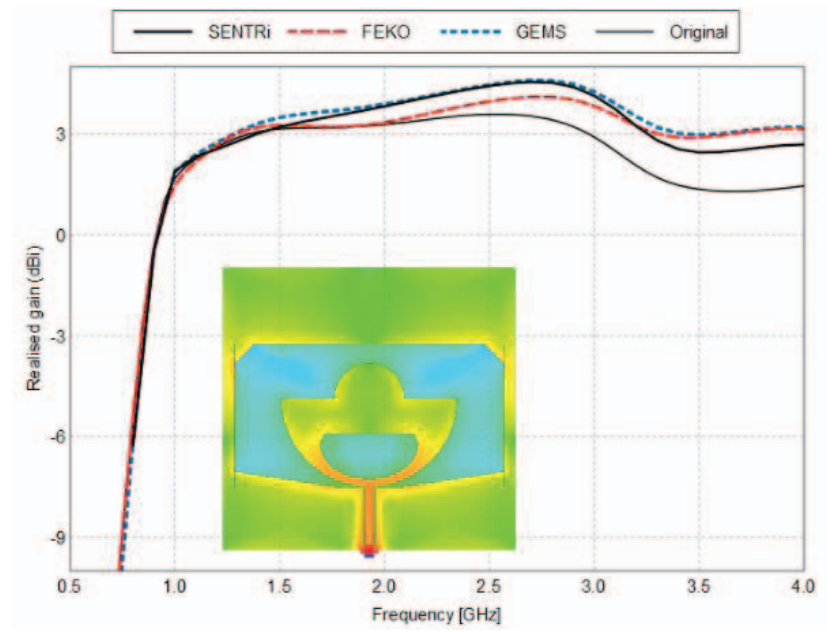

(b)

Fig. 3. Modified planar slot antenna: (a) reflection coefficient and (b) boresight gain vs. frequency.

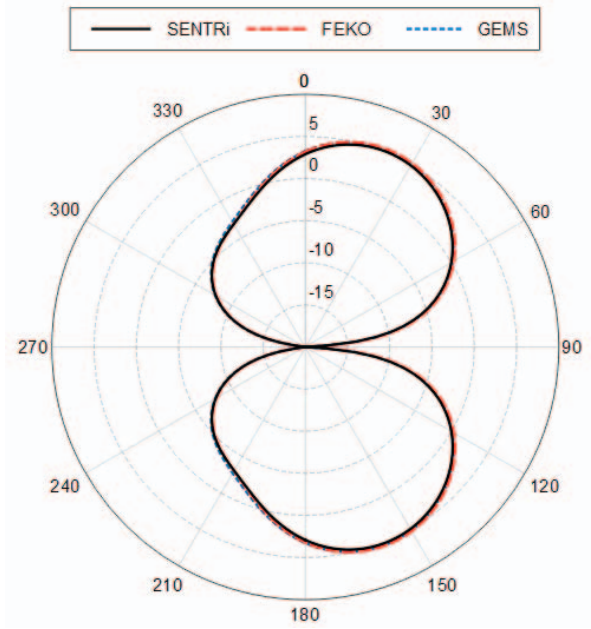

Fig. 4. Planar slot antenna E-plane radiation pattern at $4 \mathrm{GHz}$.

\section{CONCLUSION}

Ground penetrating radars typically operate over a decade IBW with antennas in close proximity to the soil. The UWB antenna is a critical component and should radiate a sharp pulse with low reverberation (ring down) from multiple reflections over the antenna structure [3]. The design objective is complicated by physically (and electrically) small antenna requirements for hand-held systems. Simulation results indicate that the slotted radiator can improve the gain at zenith by about $0.5 \mathrm{dBi}$. This seems like a marginal improvement but uniform gain is desired. The revised antenna has not been fully explored or optimized to further improve the gain vs. frequency. The codes produce similar results indicating that this antenna would require lossy dielectric loading to meet the low frequency requirement. This is common practice to sacrifice antenna efficiency for broad IBW and flat gain response over the required frequency range.

\section{REFERENCES}

[1] M. Koohestani and M. Golpur, "Very ultra-wideband printed CPW-fed slot antenna," Electronics Letters, vol. 45, no. 21, October 2009

[2] W. Coburn, "An ultra-wideband absorber backed planar slot antenna," in Proc. Applied Computational Electromagnetics Int. Symposium, Williamsburg VA, 2015.

[3] L. Gurel and U Oguz, "Simulation of ground-penetrating radars over lossy and heterogeneous grounds," IEEE Trans. Geosci. Remote Sensing, vol. 39, no. 6, pp. 1190-1197, June 2001. 